\title{
Resonant Raman Scattering of the Smallest Single-Walled Carbon Nanotubes
}

\author{
Z. K. Tang, ${ }^{1, *}$ J. P. Zhai,,${ }^{1,3, *}$ Y. Y. Tong, ${ }^{1}$ X. J. Hu, ${ }^{2}$ R. Saito, ${ }^{4}$ Y. J. Feng, ${ }^{1}$ and Ping Sheng ${ }^{1}$ \\ ${ }^{1}$ Department of Physics, Hong Kong University of Science and Technology, Clear Water Bay, Kowloon, Hong Kong \\ ${ }^{2}$ Department of Chemical Engineering, Hong Kong University of Science and Technology, Clear Water Bay, Kowloon, Hong Kong \\ ${ }^{3}$ College of Electronic Science and Technology, Shenzhen University, Shenzhen, China \\ ${ }^{4}$ Department of Physics, Tohoku University and CREST, JST, Sendai 980-8578, Japan
}

(Received 18 January 2008; published 25 July 2008)

\begin{abstract}
We report optical properties of the smallest single-walled carbon nanotubes (SWNTs) with a diameter of only $3 \AA$ A. These ultrasmall SWNTs are fabricated in the elliptical nanochannels of an AlPO-11 (AEL) single crystal. Polarized and resonant Raman scattering unambiguously revealed that these $0.3 \mathrm{~nm}$ SWNTs are of $(2,2)$ armchair symmetry. Interestingly, the $(2,2)$ armchair tube has two metastable ground states corresponding to two slightly different lattice constants in the axial direction: one state is metallic and the other is semiconducting.
\end{abstract}

DOI: 10.1103/PhysRevLett.101.047402

Single-walled carbon nanotubes (SWNTs) [1,2] have attracted extensive attention owing to their novel electronic and mechanical properties [3]. In recent years, efforts have been made to produce ultrasmall carbon nanotubes. Qin et al. and Zhao et al. observed nanotubes with a diameter of only $0.4 \mathrm{~nm}$ [4] and $0.3 \mathrm{~nm}$ [5], respectively, as an innermost layer inside multiwalled carbon nanotubes. Using an aberration-corrected high-resolution transmission electron microscopy, the chirality structure of these smallest carbon nanotubes can even be unambiguously identified [6]. Only small SWNTs show superconductivity [7-10] because of the enhancement of electron-phonon coupling due to strong curvature $[7,8]$, and the enhancement of superconducting correlations due to the strong screening of the long-range part of Coulomb repulsion [11]. However, a freestanding SWNT will eventually become unstable when its diameter is smaller than a critical value. Calculations based on energetic considerations have shown that a freestanding SWNT with a diameter of $0.4 \mathrm{~nm}$ is already marginal in stability [12-15]. Experimental observation has shown that the structure of freestanding $0.4 \mathrm{~nm}$ SWNTs in a high vacuum can be irreversibly damaged at a relatively low temperature of only $730 \mathrm{~K}$ [16], in contrast to largediameter nanotubes which are thermally stable up to 4000 K [17]. Therefore, SWNTs with a diameter smaller than $0.4 \mathrm{~nm}$ may not survive alone without the spatial confinement of outer walls.

In our previous works, we reported $0.4 \mathrm{~nm}$ SWNTs accommodated in the nanochannels of $\mathrm{AlPO}_{4}-5$ zeolite single crystals $[9,18-22]$. There are a few different types of zeolite crystals with different specific porous structures. In this Letter, we report novel electronic and optical properties of the 3D array of the smallest SWNTs fabricated in the channels of zeolite AlPO-11 (AEL) single crystals. These monosized SWNTs, with a diameter of only $0.3 \mathrm{~nm}$, are unstable in free space, but they are stable when being confined in the nanochannels. Polarized resonant Raman scattering revealed that these $0.3 \mathrm{~nm}$ SWNTs are of $(2,2)$ armchair symmetry. Interestingly,
PACS numbers: 78.67.Ch, 72.20.-i, 72.80.Jc, 78.30.Na

the $(2,2)$ SWNT has two metastable ground states corresponding to two slightly different lattice constants in the axial direction: one state is metallic and the other is semiconducting.

An AEL crystal is of Ima2 space group symmetry; its framework consists of a 10-ring elliptical channel with a cross-section dimension of $0.69 \mathrm{~nm} \times 0.91 \mathrm{~nm}[23,24]$. Figure 1(a) shows a typical scanning electron microscope image of the AEL single crystals. Its crystal framework viewed along the (001) direction is shown in Fig. 1(b). The SWNTs were synthesized by pyrolyzing dipropylamine carbon precursor molecules in the AEL nanochannels. The carbon precursor containing AEL single crystals were dehydrated at $110^{\circ} \mathrm{C}$ in a vacuum of $10^{-3}$ mbar for $2 \mathrm{~h}$ and then gradually heated up to $600{ }^{\circ} \mathrm{C}$ for $5 \mathrm{~h}$.

In Fig. 2, we show the Raman spectrum of a SWNT@AEL single crystal measured using the 514.5-nm line of an Ar$\mathrm{Kr}$ ion laser as the excitation. All Raman spectra were measured under a backscattering configuration using a microRaman system (Jobin Yvon T64000). In general, the Raman spectrum exhibits two main features: (1) in the lowfrequency region $\left(300-1100 \mathrm{~cm}^{-1}\right)$, there exists a strong characteristic radial-breathing-mode (RBM) at $760 \mathrm{~cm}^{-1}$ together with some weak features; (2) the tangential graphitelike $G$ band is split into $G^{+}$modes in the high frequency region (1550-1620 $\mathrm{cm}^{-1}$ ) and $G^{-}$modes in the intermediate frequency region $\left(1110-1500 \mathrm{~cm}^{-1}\right)$. Usually, a defect or disorder-induced $D$ band also appears in the intermediate frequency region. We prefer to attribute the Raman bands centered at $1200 \mathrm{~cm}^{-1}\left(G_{M}{ }^{-}\right)$and $1400 \mathrm{~cm}^{-1}\left(G_{S}{ }^{-}\right)$ to the $G^{-}$modes because of the following two facts. First, the frequencies of these bands are independent of excitation photon energy, as shown in the inset of Fig. 2, in contrast to the significant dependence of the $D$ mode frequency on the excitation photon energy. Second, no obvious Raman features were observed in the double-frequency region, in contrast to the intense Raman signal in the $2 D$ frequency region usually accompanying the $D$ mode $[25,26]$. 

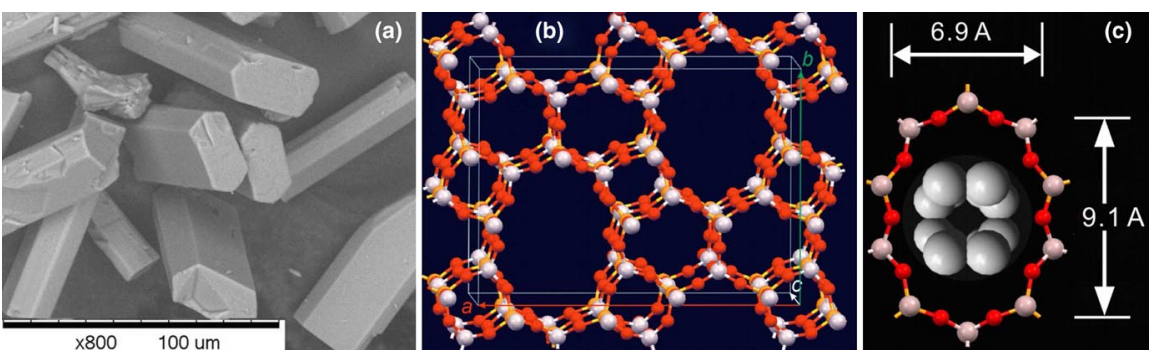

FIG. 1 (color online). (a) Scanning electron microscope image of AEL single crystals. (b) The crystal structure. The dark spheres represent oxygen atoms and the light spheres denote $\mathrm{Al}$ or $\mathrm{P}$ atoms. (c) The 10-ring channel, viewed along (001) direction. An armchair $(2,2)$ carbon nanotube is also sketched inside.
Considering the van der Waals radius of the carbon atom and the dimension of the elliptical channel, the diameter of the nanotube which could be accommodated in the channel would not be larger than $0.3 \mathrm{~nm}$. There are three possible structures for SWNTs with a diameter of about $0.3 \mathrm{~nm}$; they are the armchair $(2,2)$, the chiral $(3,1)$, and the zigzag $(4,0)$. To understand the Raman modes, we calculated vibration modes for the possible three tube structures based on a density function approximation. In the calculation, we have set the cutoff energy of the plane wave expansion at $700 \mathrm{eV}$, and applied a $5 \times 5 \times 10 k$-point grid on the reciprocal unit cell. The atoms in the unit cells were fully relaxed until the force acting on each atom was less than $0.01 \mathrm{eV} / \AA$. Among the three structures, the $(4,0)$ tube has the largest optimized diameter $(3.32 \AA)$, followed by the $(3,1)$ tube $(3.02 \AA)$ and the $(2,2)$ tube $(2.81 \AA)$. The difference in their formation energies is relatively small: the $(4,0)$ tube has the lowest energy, with $0.15 \mathrm{eV} /$ atom higher for the $(3,1)$ tube and further $0.12 \mathrm{eV} /$ atom higher for the $(2,2)$ tube, respectively. The vibration frequencies of the characteristic RBM of the $(4,0)$, the $(3,1)$, and the $(2,2)$ are $620 \mathrm{~cm}^{-1}, 663 \mathrm{~cm}^{-1}$, and $760 \mathrm{~cm}^{-1}$, respectively. Our calculation result is consistent with those reported in the literature [5,27-29]. The RBM frequency observed in the Raman spectrum (Fig. 2) agrees excellently with that calculated for the $(2,2)$ tube. Thus, we conclude that the $(4,0)$ and the $(3,1)$ might be too large to be comfortably hosted in the AEL elliptical channels, and the armchair $(2,2)$ is the only possible structure. The structure of the $(2,2)$ tube inside the 10-ring channel is sketched in Fig. 1(c). The other Raman-active modes in the lowfrequency region calculated for the $(2,2)$ tube are shown by the vertical bars at the bottom of Fig. 2. These calculated Raman-active modes are also in good agreement with the frequencies observed in the Raman spectrum, which further confirmed that the armchair $(2,2)$ structure is the only type of SWNT accommodated in the AEL channels.

Figures 3(a) and 3(b) display Raman spectra of the $\mathrm{G}^{+}$ band measured at three polarization configurations detected under the laser excitation perpendicular to [Fig. 3(a)] and along [Fig. 3(b)] the tube direction, respectively. The capital letters shown in the figure indicate the polarization directions of the (incident, scattered) light beams; the SWNTs are orientated along the $Z$ direction. The $G^{+}$band is essentially decomposed into three bands, $A_{1 g}, E_{1 g}$, and $E_{2 g}$. When the laser excitation is perpendicular to the tube direction [Fig. 3(a)], at the $Z Z$ polarization configuration, all these three bands are clearly observed in the spectrum, dominated by the $A_{1 g}$ mode. At the $X X$ configuration, the spectrum is dominated by the $A_{1 g}$ and $E_{2 g}$ modes with a more significant contribution from $E_{2 g}$, and the $E_{1 g}$ mode becomes negligibly weak. In comparison with the spectra in Fig. 3(a), the Raman signals of the same modes measured under perpendicular excitation [Fig. 3(b)] reveals several significant differences: (1) the $A_{1 g}$ and $E_{2 g}$ modes dominate the spectra in all three polarization configurations with only a negligibly small contribution of the $E_{1 g}$ mode, and (2) the line shape and intensity of the $G^{+}$ band are nearly polarization independent. This polarization dependence is consistent with the symmetry nature of these tangential vibrating modes. The complete angular dependences of the intensities $I(\theta)$ of the $A_{1 g}$ modes at $1591 \mathrm{~cm}^{-1}$ are shown in Fig. 3 for the $V V$ configuration (parallel polarization of incident laser and Raman signal), for the perpendicular $[\mathrm{P}(\mathrm{a})]$ and parallel $[\mathrm{P}(\mathrm{b})]$ excitations, respectively. Here the polarizations at 0 (180) and 90 (270) degrees correspond to the $Z Z$ (or $Y Y$ ) and $X X$ configurations in (a) and (b). When the excitation laser beam is incident perpendicular to the nanotube direction, the

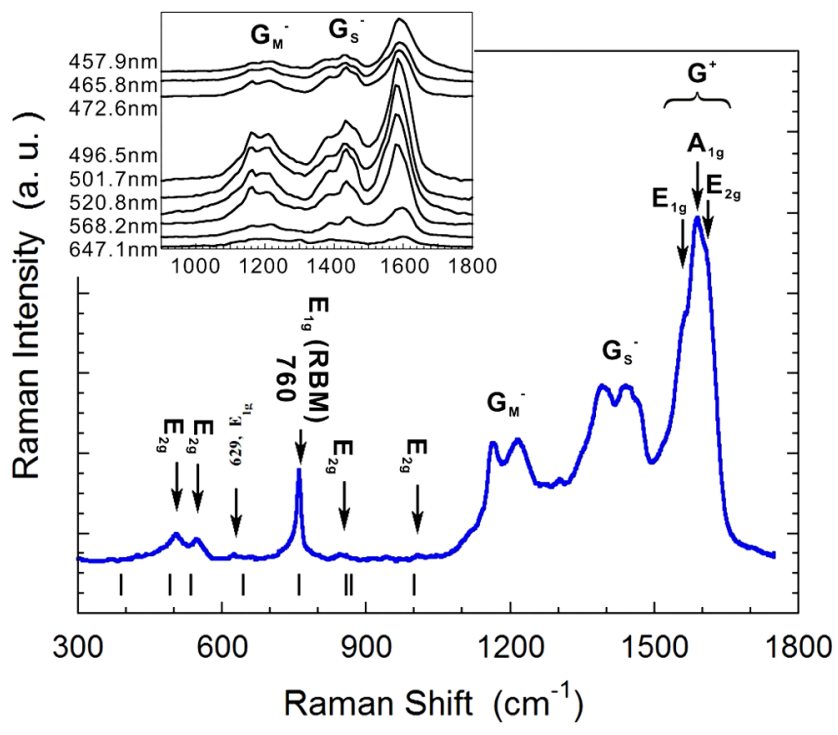

FIG. 2 (color online). Raman spectrum of a SWNT@AEL single crystal. The vertical bars at the bottom are frequencies of the Raman-active modes for the $(2,2)$ armchair SWNT calculated using a density functional approximation. The Raman spectra measured using different excitation laser wavelengths are plotted in the inset. 


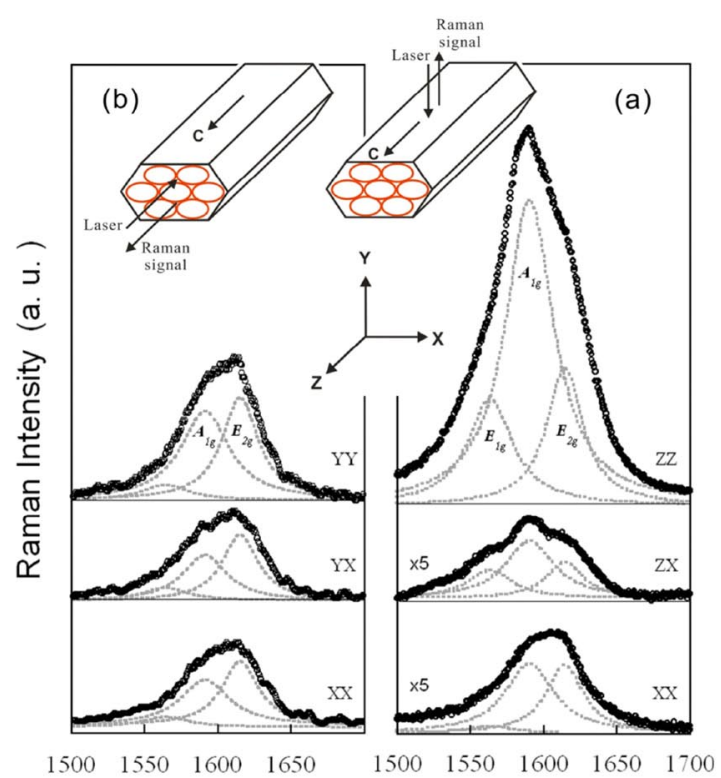

Raman Shift $\left(\mathrm{cm}^{-1}\right)$

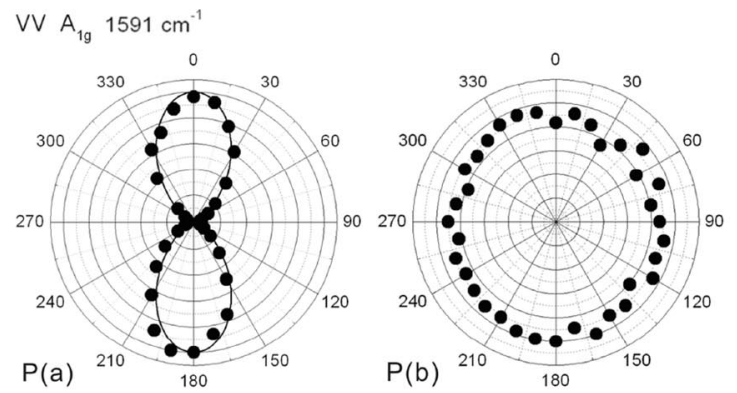

FIG. 3 (color online). Polarized Raman spectra in the $G^{+}$ band region collected under (a) perpendicular excitation and (b) parallel excitation in three polarization configurations. Polar diagrams in the lower panel show the Raman intensities of the $A_{1 g}$ mode (solid circles) as a function of the angle $\theta$ between the polarization of the incident light and the nanotube axis under the $V V$ polarization configuration measured at an excitation laser beam perpendicular $[P(a)]$ to and along $[P(b)]$ the tube direction.

Raman intensity can be described well by $I(\theta) \propto \cos ^{4}(\theta)$ for the $V V$ configuration and $I(\theta) \propto \cos ^{2}(\theta) \sin ^{2}(\theta)$ for the $V H$ (perpendicular polarization of incident laser and Raman signal) configuration (not shown in the figure). This Raman polarization effect can be attributed to electronic resonance effects and depolarization effects caused by the strongly anisotropic geometry of the nanotubes [3033]. When the excitation laser is incident along the tube direction, the Raman intensities are almost isotropic, as shown by $\mathrm{P}(\mathrm{b})$ in the lower panel, further reflecting the symmetry nature of the vibration mode.

To reveal the electronic band structure, we have studied the resonant Raman spectra for the $0.3 \mathrm{~nm}$ SWNTs. Figure 4(a) shows the Raman spectra in the low-frequency region (400-800 $\mathrm{cm}^{-1}$ ) excited using laser lines of different photon energies. The spectra are baseline corrected and normalized to the laser power. A remarkable characteristic is the strong dependence of the Raman intensity on the excitation laser wavelength. With the decrease in laser wavelength, the Raman intensity increases gradually, and the maximum Raman intensity is found at laser wavelengths around $472 \mathrm{~nm}(2.62 \mathrm{eV})$. For shorter laser wavelengths, the Raman intensity drops again. The integrated Raman intensities of the $A_{1 g}$ mode (RBM) at $760 \mathrm{~cm}^{-1}$, and the $E_{2 g}$ modes at $502 \mathrm{~cm}^{-1}$ and $547 \mathrm{~cm}^{-1}$, are plotted in Fig. 4(b) as a function of the excitation photon energy. The similar intensity profile of these Raman modes indicates that they result from the same origin. The Raman signals are obviously resonance enhanced at two particular excitation energies: a dominating peak at $2.6 \mathrm{eV}$ and a weak peak at $2.2 \mathrm{eV}$.

The band structure that we calculated based on the density function approximation for the $(2,2)$ nanotube with a regular lattice constant, $c=2.485 \AA$, is same as that reported in Ref. [34]. Interestingly, in our calculation we found that the $(2,2)$ nanotube is of two metastable ground states at slightly different lattice constants in the tube direction. Figure 4(c) shows the total energy of the $(2,2)$ tube unit cell plotted as a function of the lattice constant along the $c$ axis. The two local minima appear at $c=2.485 \AA$ and $2.580 \AA$, respectively. The tube of $c=$ $2.580 \AA$ is an indirect narrow-gap semiconductor, while the tube of $c=2.485 \AA$ is metallic. Such a double-ground state is rarely found in other carbon nanotubes. The cause of this unique property can be traced to the intrinsic bonding behavior of the carbon atoms. The competition between the energies of the bonds along the tube and that perpendicular to the tube gives rise to the two ground state structures. For the $c=2.485 \AA$ tube, the structural relaxation brings an overlap at the Fermi energy between valence and conduction bands. Upon relaxation, one of the bands from the valence region is systematically shifted up and two of the conduction bands are shifted down towards the Fermi energy and cross the valence bands, making the tube metallic [34]. For the $c=2.580 \AA$ tube, on the other hand, the electrons are more localized at the bonds perpendicular to the tube axis; as a result, an indirect narrow gap is opened. In Fig. 4(d), we show dielectric functions calculated for the $(2,2)$ carbon tube corresponding to the two band structures. We note that the two peaks of the resonant Raman spectrum are well represented in the calculated dielectric functions, implying that the semiconducting and metallic $(2,2)$ nanotubes are coexisting in the AEL channels. If the intensity of the Raman signal is proportional to the population of nanotubes, then Fig. 4(b) implies that the population of the semiconducting $(2,2)$ tubes is higher than that of metallic $(2,2)$ tubes. This is reasonable, as the total formation energy of the semiconducting $(2,2)$ tube is lower than that of the metallic $(2,2)$ tube [Fig. 4(c)]. It is worthwhile to point out that in such a small SWNT, the exciton effect would dominate over interband transition [35-37]. For that reason, the measured spectra should 

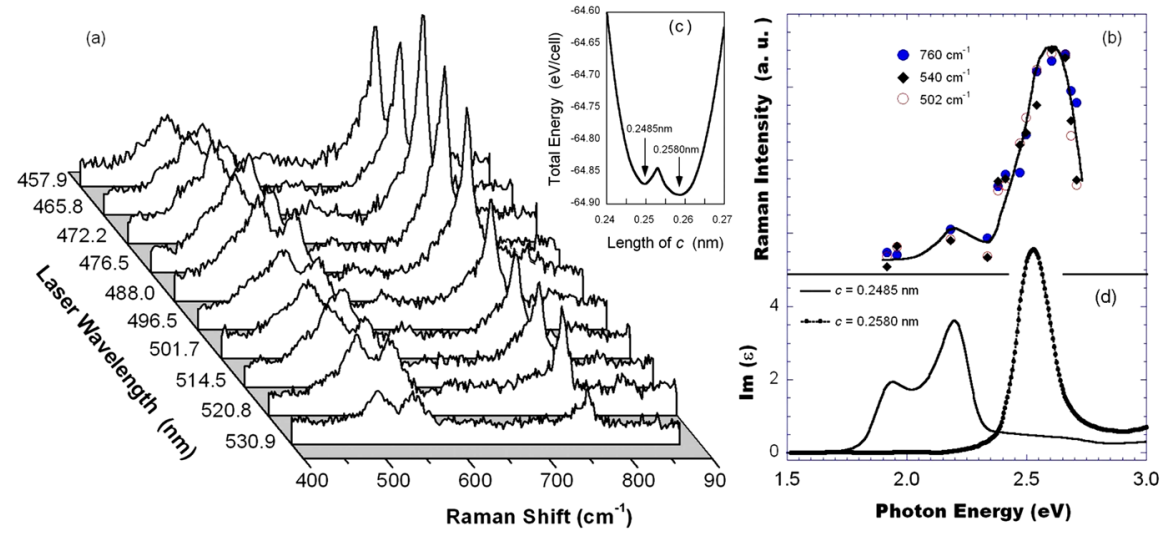

FIG. 4 (color online). (a) Raman spectra in the low-frequency region excited using different laser lines. (b) The integrated resonant Raman intensity plotted as a function of excitation photon energy. (c) The calculated total energy of the $(2,2)$ nanotube plotted as a function of $c$-axis lattice constant. (d) The calculated dielectric functions of the $(2,2)$ carbon nanotube corresponding to the two ground state structures of $c=2.485 \AA$ (solid line) and $c=2.580 \AA$ (dotted line), respectively. correspond to exciton spectra for a quantitative comparison. Our first principles calculation cannot produce the exciton binding energy. However, in such a small nanotube, the cancellation effect between the exciton binding energy and the self-energy should be obvious [38]. Thus the energy difference between the excitonic transition and interband transition should not be a critical issue in this energy scale we are considering.

By keeping in mind the dual electronic structures of the $(2,2)$ nanotubes, let us look at the $G_{M}{ }^{-}$and $G_{S}{ }^{-}$(Fig. 2) in more detail. The frequency of the $G^{-}$mode is given approximately by an empirical equation, $\omega_{G^{-}}=\omega_{G^{+}}-$ $C_{i} / d^{1.4}$; here $C_{i}$ is a constant [24,25], where $C_{\text {metal }}=$ $79.5 \mathrm{~cm}^{-1} \mathrm{~nm}^{1.4}$ and $C_{\text {semiconduct }}=47.7 \mathrm{~cm}^{-1} \mathrm{~nm}^{1.4}$. Using a diameter of $0.3 \mathrm{~nm}$ and the above $C_{i}$, the calculated frequencies of the $G^{-}$modes are $1163 \mathrm{~cm}^{-1}$ for the metallic $(2,2)$ tube and $1334 \mathrm{~cm}^{-1}$ for the semiconducting $(2,2)$ tube. These values approximately agree with the metallic $G_{M}^{-}$band (centered at $1200 \mathrm{~cm}^{-1}$ ) and semiconducting $G_{S}^{-}$band (centered at $1400 \mathrm{~cm}^{-1}$ ) shown in Fig. 2.

In summary, ultrathin SWNTs with a diameter of only $0.3 \mathrm{~nm}$ were synthesized in the AEL nanochannels. The $0.3 \mathrm{~nm}$ SWNT is of the $(2,2)$ armchair structure. Interestingly, the $(2,2)$ nanotube has two metastable ground states, one being metallic and the other being semiconducting. From our phonon dispersion calculation, we have good reason to believe that complex relaxations can lead to lower energy states with big unit cells.

We thank Professor C. T. Chan for his stimulating discussion and help in the $a b$ initio calculation. This research was supported by Hong Kong CERGC Grants No. 602807 and No. 603108, RGC Central Allocation No. CA04/ 05.SC02, and HKUST President Direct Allocation No. F0204-A. R.S. acknowledges the MEXT Grant (No. 16076201).

*Corresponding authors. phzktang@ust.hk; jpzhai@szu.edu.cn

[1] D. S. Bethune et al., Nature (London) 363, 605 (1993).

[2] S. Iijima and T. Ichihashi, Nature (London) 363, 603 (1993).
[3] See, for example, R. Saito, G. Dresselhaus, and M.S. Dresselhaus, Physical Properties of Carbon Nanotubes (Imperial College Press, London, 1998).

[4] L. Qin et al., Nature (London) 408, 50 (2000).

[5] X. Zhao et al., Phys. Rev. Lett. 92, 125502 (2004).

[6] L. Guan, K. Suenaga, and S. Iijima, Nano Lett. 8, 459 (2008).

[7] L. X. Benedict et al., Phys. Rev. B 52, 14935 (1995).

[8] K. Sasaki et al., J. Phys. Soc. Jpn. 76, 033702 (2007).

[9] Z. K. Tang et al., Science 292, 2462 (2001).

[10] M. Kociak et al., Phys. Rev. Lett. 86, 2416 (2001).

[11] S. Bellucci et al., Physica C (Amsterdam) 460-462, 1057 (2007).

[12] S. Sawada and N. Hamada, Solid State Commun. 83, 917 (1992).

[13] J. Tersoff, Phys. Rev. Lett. 61, 2879 (1988).

[14] I. Cabria, J. W. Mintmire, and C. T. White, Int. J. Quantum Chem. 91, 51 (2003); Phys. Rev. B 67, 121406 (2003).

[15] A. A. Lucas, P. H. Lambin, and R. E. Smalley, J. Phys. Chem. Solids 54, 587 (1993).

[16] J. T. Ye and Z. K. Tang, Phys. Rev. B 72, 045414 (2005).

[17] K. Metenier et al., Carbon 40, 1765 (2002).

[18] Z. K. Tang et al., Appl. Phys. Lett. 73, 2287 (1998).

[19] N. Wang, Z. K. Tang, G. D. Li, and J.S. Chen, Nature (London) 408, 50 (2000).

[20] Z. K. Tang et al., New J. Phys. 5, 146 (2003).

[21] J. P. Zhai et al., J. Phys. Chem. B 110, 19285 (2006).

[22] J. P. Zhai et al., Phys. Status Solidi B 243, 3082 (2006).

[23] F. Y. Jiang et al., J. Cryst. Growth 283, 108 (2005).

[24] J. P. Zhai, Ph.D. thesis, Hong Kong University of Science and Technology, 2007.

[25] A. Jorio et al., Phys. Rev. B 65, 155412 (2002).

[26] A. Jorio et al., New J. Phys. 5, 139 (2003).

[27] Y.L. Mao et al., Nanotechnology 15, 1000 (2004).

[28] L. M. Peng et al., Phys. Rev. Lett. 85, 3249 (2000).

[29] N. Sano et al., Phys. Rev. B 66, 113403 (2002).

[30] H. H. Gommans et al., J. Appl. Phys. 88, 2509 (2000).

[31] A. M. Rao et al., Science 275, 187 (1997).

[32] G. S. Duesberg et al., Phys. Rev. Lett. 85, 5436 (2000).

[33] A. Jorio et al., Phys. Rev. Lett. 90, 107403 (2003).

[34] R. Scipioni, Phys. Status Solidi B 244, 3137 (2007).

[35] C. D. Spataru et al., Phys. Rev. Lett. 92, 077402 (2004).

[36] V. Perebeinos, J. Tersoff, and Ph. Avouris, Phys. Rev. Lett. 92, 257402 (2004).

[37] E. Chang et al., Phys. Rev. Lett. 92, 196401 (2004).

[38] K. Sato et al., Phys. Rev. B 76, 195446 (2007). 